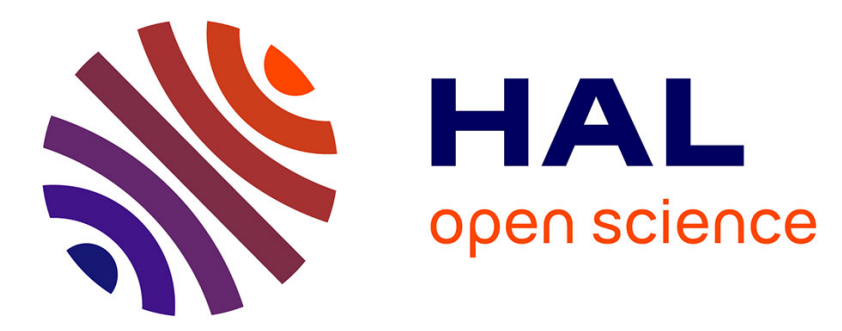

\title{
Caractérisation, sous contraintes mécaniques, de rubans ferromagnétiques amorphes MG 2605 SC, MG 2605 S3, MG $2705 \mathrm{M}$
}

J. Decalonne, J.C. Faugières, J.F. Rialland, Raymonde Bonnefille

\section{- To cite this version:}

J. Decalonne, J.C. Faugières, J.F. Rialland, Raymonde Bonnefille. Caractérisation, sous contraintes mécaniques, de rubans ferromagnétiques amorphes MG 2605 SC, MG 2605 S3, MG 2705 M. Revue de Physique Appliquée, 1985, 20 (10), pp.681-687. 10.1051/rphysap:019850020010068100 . jpa00245383

\section{HAL Id: jpa-00245383 \\ https://hal.science/jpa-00245383}

Submitted on 1 Jan 1985

HAL is a multi-disciplinary open access archive for the deposit and dissemination of scientific research documents, whether they are published or not. The documents may come from teaching and research institutions in France or abroad, or from public or private research centers.
L'archive ouverte pluridisciplinaire HAL, est destinée au dépôt et à la diffusion de documents scientifiques de niveau recherche, publiés ou non, émanant des établissements d'enseignement et de recherche français ou étrangers, des laboratoires publics ou privés. 


\title{
REVUE DE PHYSIQUE APPLIQUÉE
}

Revue Phys. Appl. 20 (1985) 681-687

OCTOBRE 1985, PAGE 681

Classification

Physics Abstracts

$75.50 \mathrm{~K}$

\section{Caractérisation, sous contraintes mécaniques, de rubans ferromagnétiques amorphes MG 2605 SC, MG 2605 S3, MG 2705 M}

\author{
J. Decalonne, J. C. Faugières, J. F. Rialland et R. Bonnefille, \\ CNAM, Laboratoire d'Electricité Industrielle, 292, rue Saint Martin, 75141 Paris Cedex 03, France
}

(Reçu le 19 avril 1985, révisé le 17 juin, accepté le 21 juin 1985)

\begin{abstract}
Résumé. - Quelques propriétés magnétiques de rubans MG 2605 SC, MG 2605 S3 et MG 2705 M sont mesurées, en fonction de contraintes de traction appliquées, dans un domaine de fréquences comprises entre $10 \mathrm{~Hz}$ et $10 \mathrm{kHz}$. Chaque ruban est soumis à plusieurs traitements thermiques afin d'obtenir des structures magnétiques sensiblement différentes. En ce qui concerne les rubans bruts de trempe, les résultats montrent l'importance de la magnétostriction responsable de l'évolution de la perméabilité d'impédance avec la contrainte exercée. L'action de l'effort de traction sur les pertes est différente selon la fréquence. A $10 \mathrm{kHz}$, celles-ci, associées principalement aux pertes par courants de Foucault, sont surtout sensibles à la taille des domaines magnétiques. Dans le cas des matériaux recuits, la structure magnétique induite a un rôle prépondérant, à basse fréquence, sur l'évolution des propriétés avec la contrainte exercée. A plus haute fréquence, les différences selon la nature du recuit sont moins prononcées.
\end{abstract}

\begin{abstract}
Some magnetic properties of amorphous ribbons MG 2605 SC, MG 2605 S3, MG 2705 M are measured over a frequency range $10 \mathrm{~Hz}-10 \mathrm{kHz}$ under applied tensile stresses. Samples are annealed with different processes to induce some change in magnetic structure. For as-cast ribbons, results exhibit the magnetostrictive effect on change in AC permeability with tensile load. The influence of the applied stress on total losses is quite different according to frequency. At $10 \mathrm{kHz}$, losses are controlled by eddy current losses and are mainly sensitive to domain size. For annealed samples, the influence in the low frequency range of tensile load on magnetic properties depends on the magnetic structure induced by the thermal process. For higher frequencies, the effects of annealing tend to vanish.
\end{abstract}

\section{Introduction.}

La résistance mécanique élevée des alliages ferromagnétiques amorphes est favorable à leur utilisation dans des conditions de contraintes sévères susceptibles d'intervenir, notamment, dans les machines électriques à grande vitesse. Cependant, les efforts auxquels ils sont soumis peuvent modifier les caractéristiques magnétiques de ces matériaux. Ainsi, les pertes électromagnétiques, dont les faibles valeurs représentent l'atout essentiel des ferromagnétiques amorphes, sont, en général, très sensibles à la présence de contraintes appliquées.

Cet article décrit l'effet des contraintes de traction longitudinale appliquées sur des rubans, fabriqués par Allied Chemical Co, de structures magnétiques sensiblement différentes. En particulier, des traitements appliqués sur un même alliage induisent, selon les cas, une anisotropie directionnelle dans le matériau $[1-5,16]$.

Les mesures sont effectuées sur des rubans unitaires (une seule couche) à l'aide d'un perméamètre décrit dans un précédent article [6]. Les caractéristiques relevées correspondent aux courbes $B(H)$ et aux pertes, dans un domaine de fréquences compris entre $10 \mathrm{~Hz}$ et $10 \mathrm{kHz}$ et pour des tractions longitudinales pouvant atteindre $60 \mathrm{daN} \cdot \mathrm{mm}^{-2}$.

La caractérisation est réalisée sous champ magnétique sinusoïdal à température ambiante. Les essais 
sous contrainte montrent l'absence d'hystérésis mécanique et de déformations irréversibles.

Seuls, les résultats les plus caractéristiques sont présentés ici. Ainsi, sont reportées, essentiellement, les évolutions de la perméabilité relative d'impédance $\mu_{\mathrm{r}}$ et des pertes électromagnétiques par unité de volume $p$ en fonction de la contrainte appliquée $\sigma$. Rappelons que la perméabilité d'impédance correspond, à une fréquence donnée, au rapport de l'amplitude de l'induction moyenne dans une section droite du ruban testé et de celle du champ magnétique en surface. Les variations de $\mu_{\mathrm{r}}$ sont tracées, dans la plupart des cas, pour une fréquence de $10 \mathrm{~Hz}$ et une induction maximale de $1 \mathrm{~T}$, celles des pertes pour $10 \mathrm{~Hz}$ et $10 \mathrm{kHz}$ et une induction encore égale à $1 \mathrm{~T}$.

\section{Résultats.}

2.1 RubaN MG $2605 \mathrm{SC}\left(\mathrm{Fe}_{81} \mathrm{~B}_{13,5} \mathrm{Si}_{3,5} \mathrm{C}_{2}\right)$. L'alliage testé se présente sous forme d'un ruban de $25 \mathrm{~mm}$ de large et $25 \mu \mathrm{m}$ d'épaisseur. Afin d'obtenir des structures magnétiques différentes, le matériau a été soumis à plusieurs traitements :

- échantillon 1 : matériau brut de fabrication, c'est-à-dire non recuit, (NR)

- échantillon 2 : ruban ayant subi un simple recuit de relaxation durant $2 \mathrm{~h}$ à $365^{\circ} \mathrm{C}(\mathrm{R})$, [7]

- échantillon 3 : ruban traité sous champ magnétique longitudinal de $800 \mathrm{~A} \cdot \mathrm{m}^{-1}$ durant $2 \mathrm{~h}$ à $350^{\circ} \mathrm{C}$ $(\mathrm{R} / \mathrm{H})$

- échantillon 4 : ruban traité sous contrainte longitudinale de $40 \mathrm{daN} . \mathrm{mm}^{-2}$ durant $2 \mathrm{~h}$ à $365^{\circ} \mathrm{C}$ $(\mathrm{R} / \sigma)$.

Tous les traitements thermiques sont effectués sous argon.

Les évolutions de $\mu_{\mathrm{r}}$ en fonction de $\sigma$ sont données sur la figure 1 ; les courbes correspondent à une fréquence de $10 \mathrm{~Hz}$ et à une induction maximale de $1 \mathrm{~T}$.

La perméabilité de l'échantillon non traité augmente jusqu'à une contrainte d'environ $5 \mathrm{daN} . \mathrm{mm}^{-2}$, puis diminue lentement avec l'accroissement de l'effort appliqué. Cette évolution peut s'expliquer par la formation, aux faibles contraintes, d'une structure en bandes due à la magnétostriction positive du matériau, puis par l'apparition de domaines plus complexes liés aux contraintes internes, associées aux fortes valeurs de traction.

Dans le cas des échantillons soumis à un recuit de relaxation et traités sous champ, les évolutions sont semblables à la précédente, avec cependant des valeurs initiales de la perméabilité $(\sigma=0)$ plus élevées et des maxima situés à environ 2 daN. $\mathrm{mm}^{-2}$. Dans le domaine des faibles contraintes, la perméabilité du matériau traité sous champ est, du fait de l'anisotropie induite, supérieure à celle de l'échantillon simplement recuit. Enfin, la perméabilité de l'échantillon traité sous contrainte diminue de façon monotone avec la traction exercée.

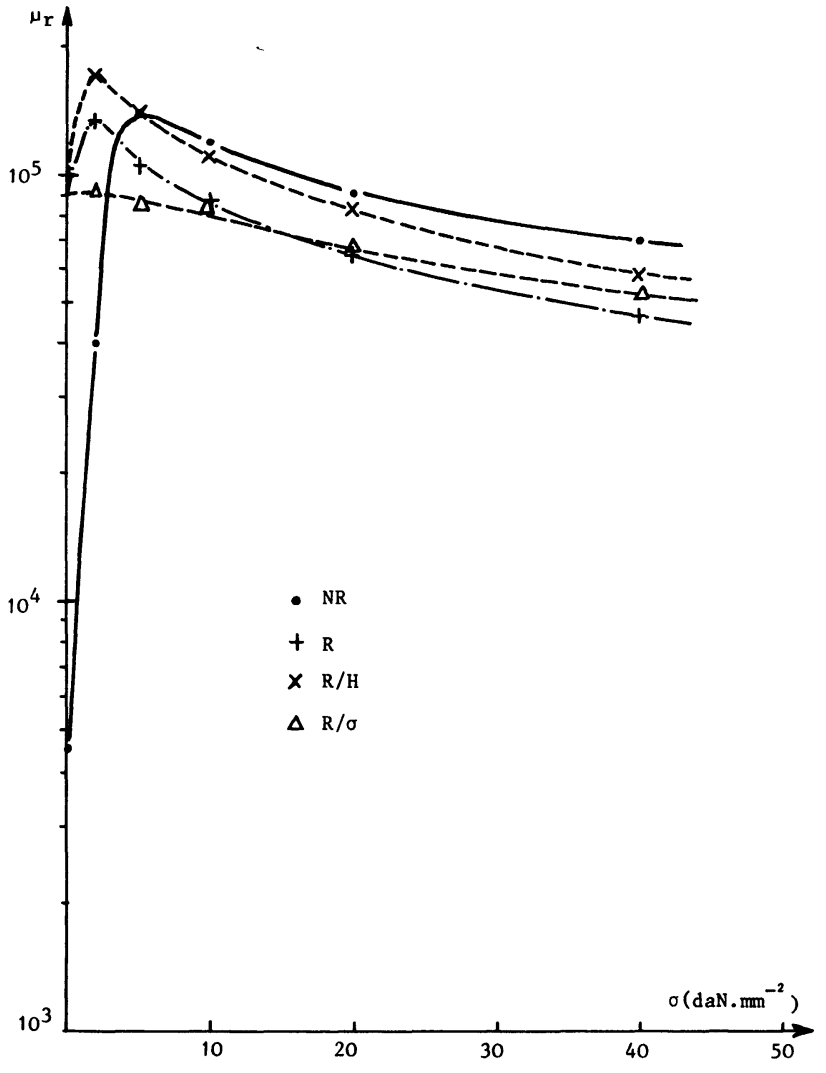

Fig. 1. - Perméabilité d'impédance en fonction de la contrainte de traction. Ruban $2605 \mathrm{SC}: B=1 \mathrm{~T}, f=10 \mathrm{~Hz}$.

[AC permeability versus applied tensile stress. Ribbon $2605 \mathrm{SC}: B=1 \mathrm{~T}, f=10 \mathrm{~Hz}$.]

Les évolutions des pertes volumiques $p$ dissipées à $10 \mathrm{~Hz}$ et $10 \mathrm{kHz}$ sont représentées sur les figures 2 et 3 .

A $10 \mathrm{~Hz}$, on note, dans le cas des échantillons naturel, recuit et traité sous champ, une diminution rapide de $p$ jusqu'à un minimum situé entre 2 et 6 daN. $\mathrm{mm}^{-2}$ suivie d'un accroissement dont la pente varie peu selon le traitement. Le comportement du ruban traité sous contrainte est différent. Jusqu'à 10 daN. $\mathrm{mm}^{-2}$, les pertes sont pratiquement constantes. Au-delà, il y a une augmentation de $p$ avec une pente comparable à celles relevées avec les autres rubans, soit environ $1,5 \%$ par daN. mm ${ }^{-2}$. En valeur absolue, les pertes du ruban traité sous contrainte sont supérieures à celles des autres échantillons pour des efforts dépassant $4 \mathrm{daN} . \mathrm{mm}^{-2}$, alors que, pour $\sigma=0$, leur valeur est inférieure.

A $10 \mathrm{kHz}$, la distinction s'établit entre le matériau naturel, pour lequel les variations sont du même type qu'à basse fréquence mais avec un minimum moins marqué, et les trois rubans traités. Ces derniers ne présentent plus qu'une croissance constante des pertes en fonction de $\sigma$, avec une pente moyenne de $1 \%$ par daN. $\mathrm{mm}^{-2}$.

De façon schématique, on peut différencier deux 


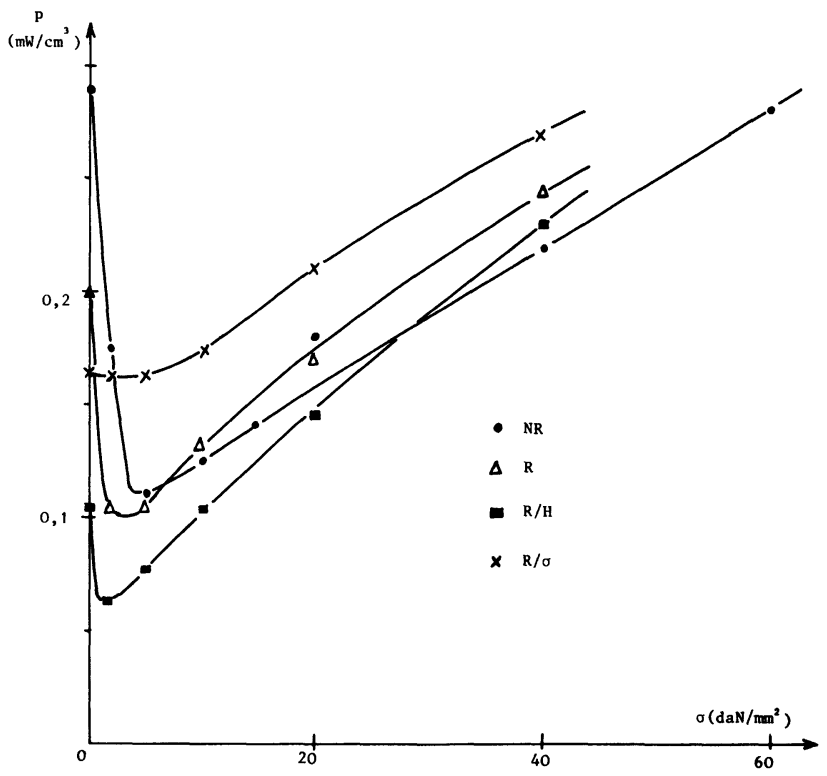

Fig. 2. - Pertes volumiques en fonction de la contrainte de traction. Ruban $2605 \mathrm{SC}: B=1 \mathrm{~T}, f=10 \mathrm{~Hz}$.

[Total losses versus $\sigma$. Ribbon $2605 \mathrm{SC}: B=1 \mathrm{~T}, f=10 \mathrm{~Hz}$.]

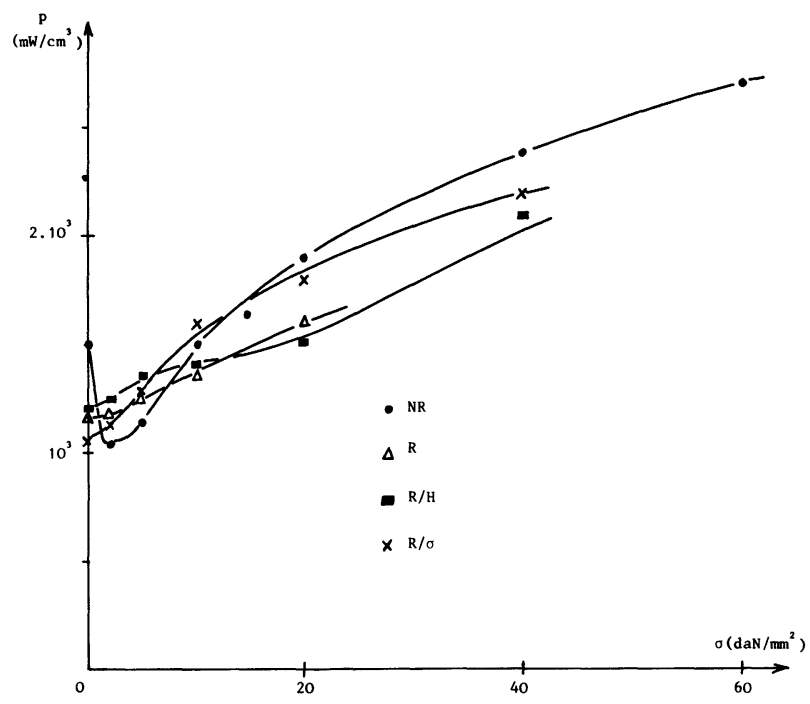

Fig. 3. - Pertes volumiques en fonction de la contrainte de traction. Ruban $2605 \mathrm{SC}: B=1 \mathrm{~T}, f=10 \mathrm{kHz}$.

[Total losses versus $\sigma$. Ribbon $2605 \mathrm{SC}: B=1 \mathrm{~T}, f=10 \mathrm{kHz}$.]

effets des efforts de traction :

- Surtout sensible aux faibles contraintes et à cause du coefficient de magnétostriction positif de l'alliage, la traction tend à créer une orientation de la structure en bandes longitudinales. Cela s'accompagne d'un accroissement de la perméabilité et d'une diminution des pertes par hystérésis. L'intensification de la contrainte entraîne un rétrécissement des domaines conduisant à la diminution des pertes par courants de Foucault [8-10]. La sensibilité du ruban à cette action dépend de sa structure initiale et, en particulier, de celle induite par le traitement.

- La seconde tendance correspond à la destruction, aux fortes contraintes, de la structure orientée acquise précédemment. Les tensions internes dues aux irrégularités d'épaisseur ou au glissement entre les fibres entraînent des structures étoilées ou circulaires [11] et cette modification impose une augmentation des pertes par hystérésis ainsi qu'une diminution de la perméabilité. Les pertes par courants de Foucault semblent peu sensibles à ce phénomène qui ne génère que des domaines de petite taille.

Afin de compléter les mesures précédentes, nous avons effectué quelques essais sur un échantillon $2605 \mathrm{SC}$ brut de trempe soumis à un effort de traction perpendiculaire à la direction du champ magnétique. Cette configuration se présente dans le cas d'un rotor à pôles lisses de machine électrique tournante.

Le ruban testé a une largeur de $10 \mathrm{~cm}$. Les mesures ont été faites à l'aide d'un perméamètre dérivé de celui utilisé pour effectuer les mesures précédentes. La précision des mesures est relativement faible, du fait des distributions non uniformes des lignes de champ et des contraintes appliquées. Seules les variations des caractéristiques peuvent être considérées comme significatives.

Le premier point à signaler est que les résultats sont identiques, que la direction du champ soit parallèle ou perpendiculaire à l'axe du ruban. Cette absence de direction préférentielle dans le plan du ruban, à l'état brut de trempe, est confirmée par une étude de la répartition des moments magnétiques par spectrométrie Mössbauer [12].

La figure 4 représente l'évolution des cycles d'hystérésis, relevés à $400 \mathrm{~Hz}$, selon que l'effort de traction est parallèle $(\sigma / / \mathbf{H})$ ou perpendiculaire $(\sigma \perp \mathbf{H})$ à la direction du champ magnétique. L'application d'une contrainte longitudinale se traduit, comme pour les essais précédents, par une augmentation de la perméabilité. Lorsque la traction est perpendiculaire au champ, la perméabilité diminue rapidement. Cet effet peut s'expliquer par la création d'une structure en bandes dirigées encore suivant la contrainte. Mais cette orientation impose une rotation des domaines pour obtenir l'aimantation du matériau suivant l'axe du champ, donc une chute de la perméabilité.

Les pertes volumiques, associées à une fréquence de $400 \mathrm{~Hz}$ et à une induction maximale de $0,7 \mathrm{~T}$, sont données sur la figure 5 . Sous contrainte longitudinale, on observe une diminution des pertes, mieux caractérisée sur les figures 2 et 3 . Sous contrainte transversale, on note un fort accroissement des pertes avec la contrainte. Cette évolution confirme l'orientation de la structure magnétique par la traction appliquée, ce qui impose, ici une rotation des domaines et donc des pertes importantes.

2.2 RubaN MG $2605 \mathrm{~S} 3\left(\mathrm{Fe}_{79} \mathrm{~B}_{16} \mathrm{Si}_{5}\right)$. - Les rubans testés, de même provenance, ont une largeur de $25 \mathrm{~mm}$ 


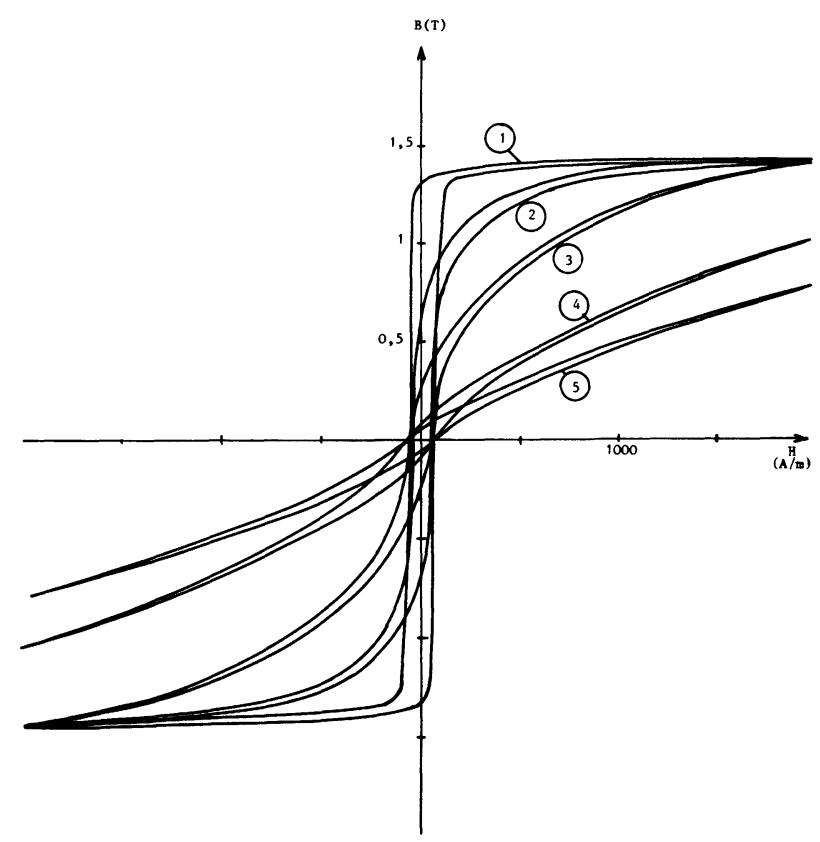

Fig. 4. - Cycles d'hystérésis relevés à $400 \mathrm{~Hz}$ en fonction de la contrainte appliquée. Ruban 2605 SC non recuit. $1: \sigma=18 \mathrm{daN} \cdot \mathrm{mm}^{-2}(\sigma / / \mathbf{H}) 2: \sigma=3 \mathrm{daN} \cdot \mathrm{mm}^{-2}$ $(\sigma / / \mathbf{H}) 3: \sigma=04: \sigma=9$ daN. $\mathrm{mm}^{-2}(\sigma \perp \mathbf{H}) 5: \sigma=$ $18 \mathrm{daN} . \mathrm{mm}^{-2}(\sigma \perp \mathbf{H})$.

[Hysteresis loop according tensile load. Ribbon 2605 SC, as-cast, $f=400 \mathrm{~Hz} .1: \sigma=18 \mathrm{daN} . \mathrm{mm}^{-2}(\sigma / / / \mathrm{H}) 2: \sigma=$ 3 daN. $\mathrm{mm}^{-2}(\sigma / / \mathbf{H}) 3: \sigma=04: \sigma=9 \mathrm{daN} \cdot \mathrm{mm}^{-2}$ $(\sigma \perp \mathbf{H}) 5: \sigma=18 \mathrm{daN} . \mathrm{mm}^{-2}(\sigma \perp \mathbf{H})$.]

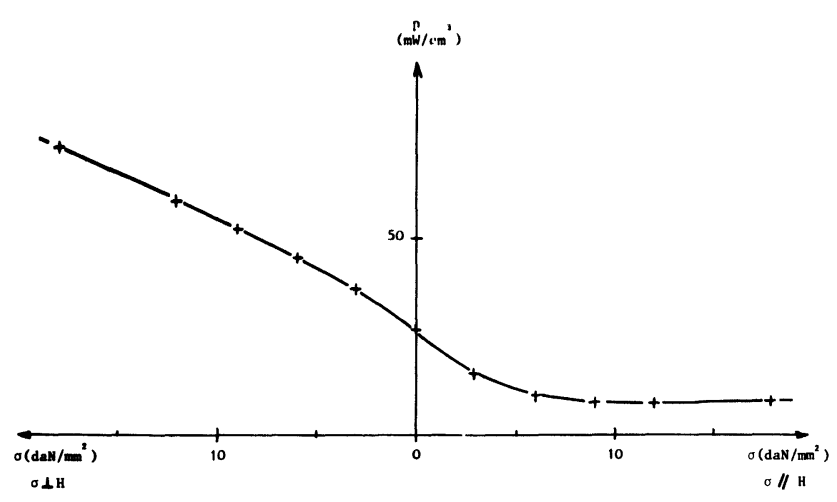

Fig. 5. - Pertes volumiques en fonction de $\sigma$. Ruban $2605 \mathrm{SC}$ non recuit : $B=0,7 \mathrm{~T}, f=400 \mathrm{~Hz}$.

[Total losses versus $\sigma$. Ribbon $2605 \mathrm{SC}$, as-cast : $B=0.7 \mathrm{~T}$, $f=400 \mathrm{~Hz}$.]

et une épaisseur de $25 \mu \mathrm{m}$. Comme précédemment, plusieurs traitements ont été effectués :

- échantillon $1:$ matériau brut de trempe (NR)

- échantillon 2 : ruban recuit à $430^{\circ} \mathrm{C}$ pendant $3 \mathrm{~h} 30$ (R)

- échantillon 3 : ruban traité sous champ longitudinal de $800 \mathrm{~A} \cdot \mathrm{m}^{-1}$ durant $3 \mathrm{~h} 30$ à $430^{\circ}(\mathrm{R} / \mathrm{H})$
- échantillon 4 : ruban recuit sous traction longitudinale de $29 \mathrm{daN} . \mathrm{mm}^{-2}$ pendant $3 \mathrm{~h} 30$ à $430{ }^{\circ} \mathrm{C}$ $(\mathrm{R} / \sigma)$.

Comme dans le cas du ruban précédent, les traitements thermiques sont effectués sous argon.

Les évolutions de la perméabilité d'impédance $\mu_{\mathrm{r}}$ en fonction de la traction longitudinale appliquée $\sigma$ sont données, pour une fréquence de $10 \mathrm{~Hz}$ et une induction maximale de $1 \mathrm{~T}$, sur la figure 6. Les courbes relatives aux échantillons 2 et 3 sont confondues; l'identité des résultats obtenus après traitements avec ou sans champ provient, sans doute, de ce que la température de recuit est supérieure à la température de Curie [7]. Le champ appliqué lors du traitement n'a alors d'action que dans la phase de refroidissement. En ce qui concerne l'échantillon brut de trempe, on constate que l'évolution de $\mu_{\mathrm{r}}$ avec $\sigma$ est semblable à celle observée avec la nuance 2605 SC (Fig. 1). Le matériau ayant encore un coefficient de magnétostriction positif, l'interprétation en est identique.

La perméabilité relative de l'échantillon traité sous champ varie de façon analogue à celle du ruban non traité. On peut cependant noter que la valeur maximale, obtenue pour une traction d'environ 2 daN. $\mathrm{mm}^{-2}$, est sensiblement inférieure à celle relative à l'échantillon brut de trempe, alors que la valeur initiale $(\sigma=0)$ est supérieure. Ce comportement est lié à la structure induite par le recuit. Celui-ci a pour rôle de créer des microprécipités dispersés dans la phase amorphe afin de réduire la taille des domaines magnétiques et, en conséquence, les pertes par courants de Foucault [13]. La présence de ces microprécipités étant défavorable à l'établissement d'une structure magnétique en bandes, il est logique que la

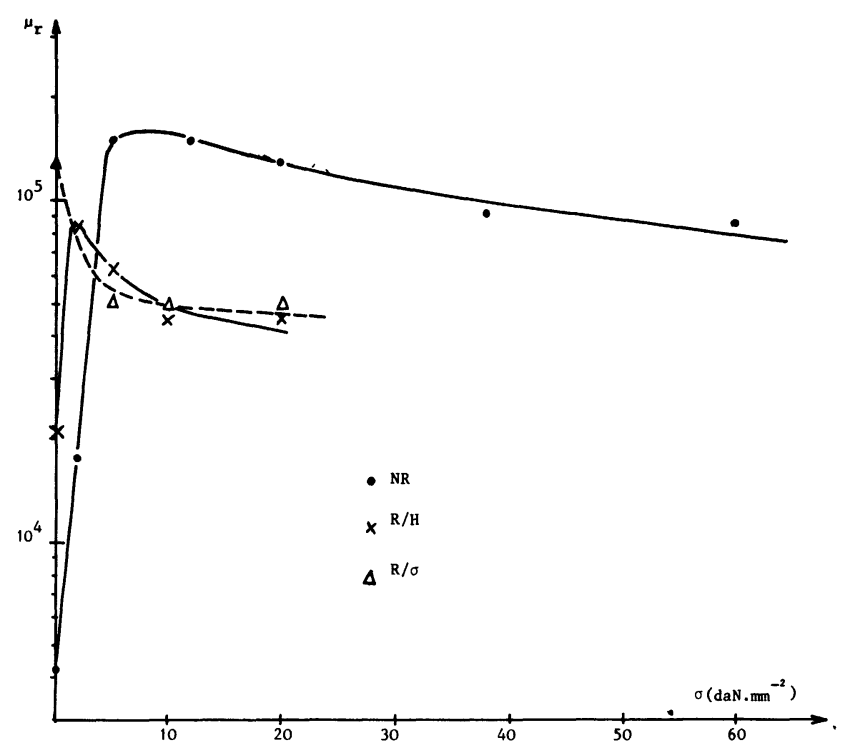

Fig. 6. - Perméabilité $\mu_{\mathrm{r}}$ en fonction de $\sigma$. Ruban $2605 \mathrm{~S} 3$ : $B=1 \mathrm{~T}, f=10 \mathrm{~Hz}$.

[AC permeability versus applied tensile stress. Ribbon $2605 \mathrm{~S} 3: B=1 \mathrm{~T}, f=10 \mathrm{~Hz}$.] 
contrainte exercée conduise à des perméabilités inférieures à celles obtenues en l'absence de cette phase dispersée. Dans le cas de l'échantillon traité sous contrainte, on note une décroissance monotone de $\mu_{\mathrm{r}}$. Hors contrainte appliquée, la valeur de $\mu_{\mathrm{r}}$ suggère l'existence d'une certaine anisotropie directionnelle induite par le traitement. Dès que $\sigma$ devient supérieur à $2 \mathrm{daN} . \mathrm{mm}^{-2}$, l'évolution de la perméabilité est pratiquement confondue avec celle du ruban traité sous champ.

Les figures 7 et 8 représentent les variations des pertes volumiques en fonction de la traction longi-

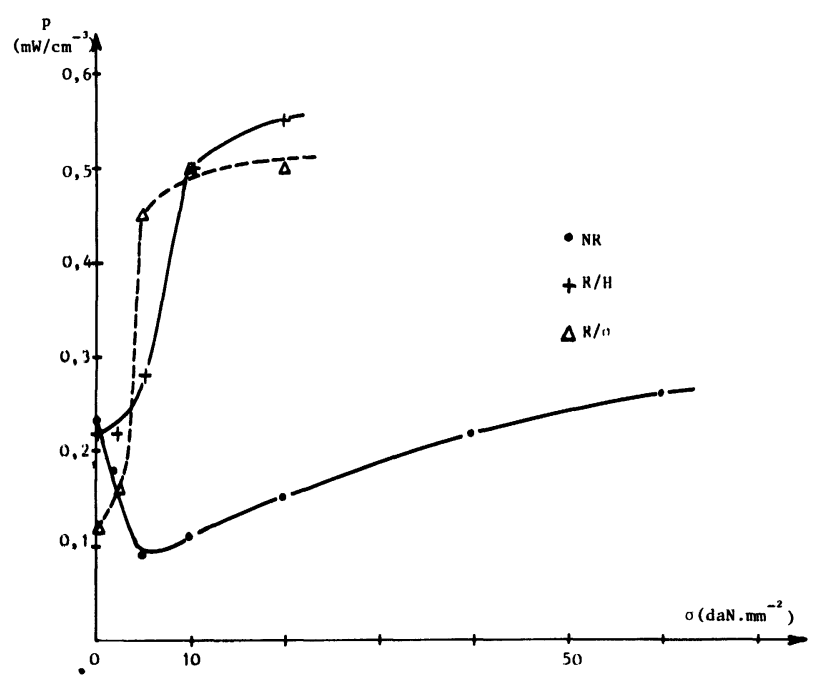

Fig. 7. - Pertes volumiques en fonction de $\sigma$. Ruban 2605 S3: $B=1 \mathrm{~T}, f=10 \mathrm{~Hz}$.

[Total losses versus $\sigma$. Ribbon $2605 \mathrm{~S} 3: B=1 \mathrm{~T}, f=10 \mathrm{~Hz}$.]

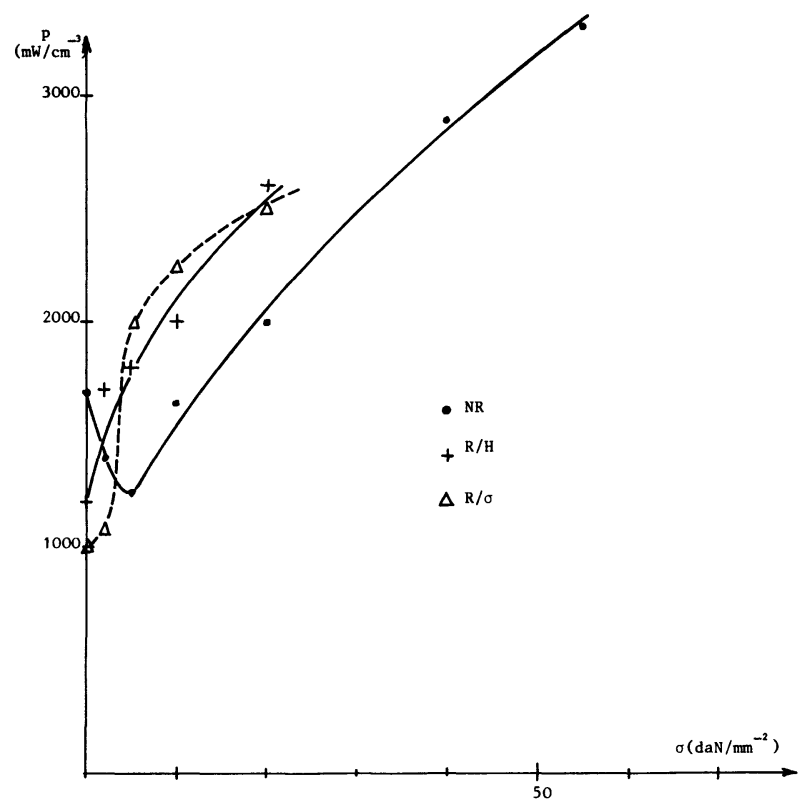

Fig. 8. - Pertes volumiques en fonction de $\sigma$. Ruban 2605 S3 : $B=1 \mathrm{~T}, f=10 \mathrm{kHz}$.

[Total losses versus $\sigma$. Ribbon $2605 \mathrm{~S} 3: B=1 \mathrm{~T}, f=10 \mathrm{kHz}$.] tudinale, exercée respectivement à $10 \mathrm{~Hz}$ et $10 \mathrm{kHz}$ Aux deux fréquences, on note que seule la courbe associée au matériau brut de trempe présente un minimum. Pour une fréquence de $10 \mathrm{~Hz}$, la plus faible valeur des pertes est obtenue, hors contrainte, avec l'échantillon 4. A cette fréquence, les pertes par hystérésis sont prépondérantes, ce qui confirme l'existence d'une anisotropie directionnelle induite par le traitement correspondant. Par contre, on observe, dès que la contrainte appliquée devient supérieure à $2 \mathrm{daN} \cdot \mathrm{mm}^{-2}$, une augmentation rapide des pertes dissipées dans les échantillons recuits par rapport à celles développées dans le matériau brut de fabrication. Ainsi, il apparaît un effet antagoniste entre l'action du microprécipité et celle de la structure magnétique induite par la traction exercée.

A plus haute fréquence $(10 \mathrm{kHz})$, l'écart entre les différentes courbes s'atténue. Cela provient de ce que les pertes correspondent alors, en grande partie, à des pertes par courants de Foucault, sensibles, en particulier à la taille des domaines. Les microprécipités, induits par les recuits, ayant pour rôle de subdiviser les domaines [13], il est clair que le traitement thermique préconisé par le fabricant a pour but de minimiser les pertes à haute fréquence. L'augmentation des pertes avec la contrainte exercée est, sans doute, due aux mêmes causes que celles à l'origine de l'accroissement de pertes dissipées, à la même fréquence, dans l'alliage 2605 SC (Fig. 3).

2.3 RubaN MG $2705 \mathrm{M}\left(\mathrm{Co}_{69} \mathrm{Fe}_{4} \mathrm{Ni}_{1} \mathrm{Mo}_{2} \mathrm{~B}_{12} \mathrm{Si}_{12}\right)$. L'alliage testé se présente sous la forme d'un ruban de $25 \mathrm{~mm}$ de large et de $25 \mu \mathrm{m}$ d'épaisseur. Le coefficient de magnétostriction à saturation de ce matériau, très riche en cobalt, est nul après traitement thermique sous champ magnétique $[7,17]$. Seulement deux échantillons ont été caractérisés :

- échantillon 1 : matériau brut de fabrication(NR)

- échantillon 2 : ruban recuit en présence d'un champ magnétique longitudinal de $800 \mathrm{~A} \cdot \mathrm{m}^{-1}$ à $315^{\circ} \mathrm{C}$ durant $20 \mathrm{~min}$. sous argon $(\mathrm{R} / \mathrm{H})$.

La figure 9 donne les variations de $\mu_{\mathrm{r}}$ avec $\sigma$, pour une fréquence de $10 \mathrm{~Hz}$ et une induction maximale de $0,5 \mathrm{~T}$. On constate que, pour $\sigma=0$, la valeur de $\mu_{\mathrm{r}}$ du ruban naturel est supérieure à celle du ruban traité sous champ. La valeur particulièrement élevée de la perméabilité, pour un matériau brut de fabrication, est due à la faible influence, sur la structure magnétique, des contraintes liées à la trempe. Lorsqu'on exerce une contrainte de traction sur l'échantillon 1 , on note une décroissance rapide de $\mu_{\mathrm{r}}$, pouvant traduire un coefficient de magnétostriction négatif. Dans le cas de l'échantillon recuit, l'influence de $\sigma$ est moins sensible; cela correspond à la diminution du coefficient de magnétostriction par le traitement thermique.

La variation des pertes, relevées à $10 \mathrm{~Hz}$, est représentée sur la figure 10. On observe que les pertes dissipées dans l'échantillon naturel augmentent avec la traction. Cela peut signifier que les domaines, relati- 


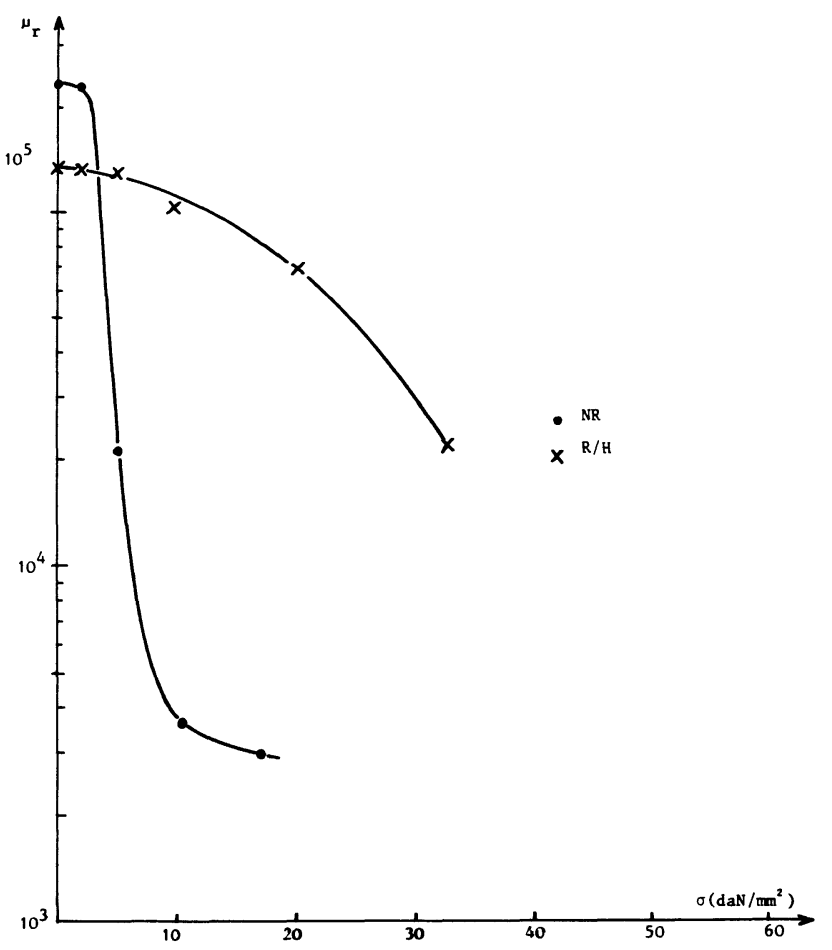

Fig. 9. - Perméabilité en fonction de $\sigma$. Ruban $2705 \mathrm{M}$ : $B=0,5 \mathrm{~T}, f=10 \mathrm{~Hz}$.

[AC permeability versus $\sigma$. Ribbon $2705 \mathrm{M}: B=0.5 \mathrm{~T}$, $f=10 \mathrm{~Hz}$.]

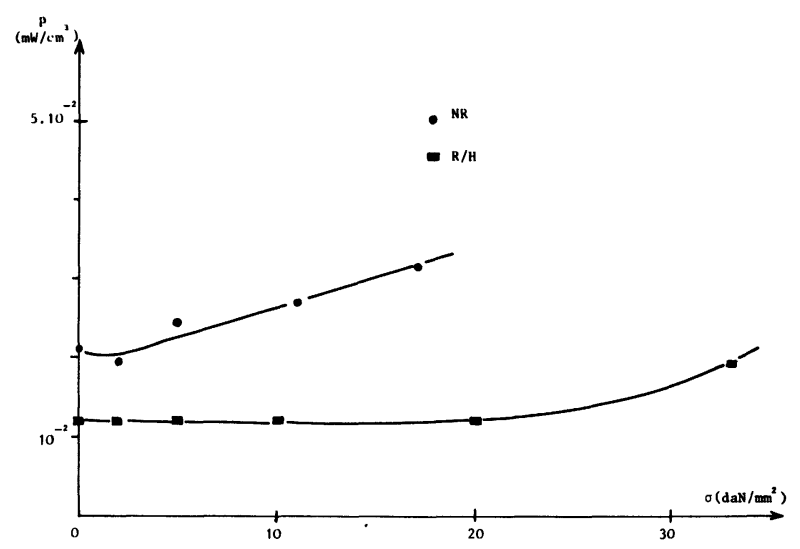

Fig. 10. - Pertes volumiques en fonction de $\sigma$. Ruban 2705 $M: B=0,5 \mathrm{~T}, f=10 \mathrm{~Hz}$.

[Total losses versus $\sigma$. Ribbon $2705 \mathrm{M}: B=0.5 \mathrm{~T}, f=10 \mathrm{~Hz}$.]

vement grands, ont tendance à s'orienter perpendiculairement à l'axe de l'effort. Par contre, les pertes associées à l'échantillon recuit sont insensibles, jusqu'à $20 \mathrm{daN} . \mathrm{mm}^{-2}$, à la contrainte exercée. Ce comportement traduit l'annulation effective du coefficient de magnétostriction. Cependant les courbes de la figure 11, sur laquelle est donnée l'évolution des pertes à $10 \mathrm{kHz}$, font apparaître que l'action de la contrainte ne peut être décrite aussi simplement que ci-dessus. En effet,

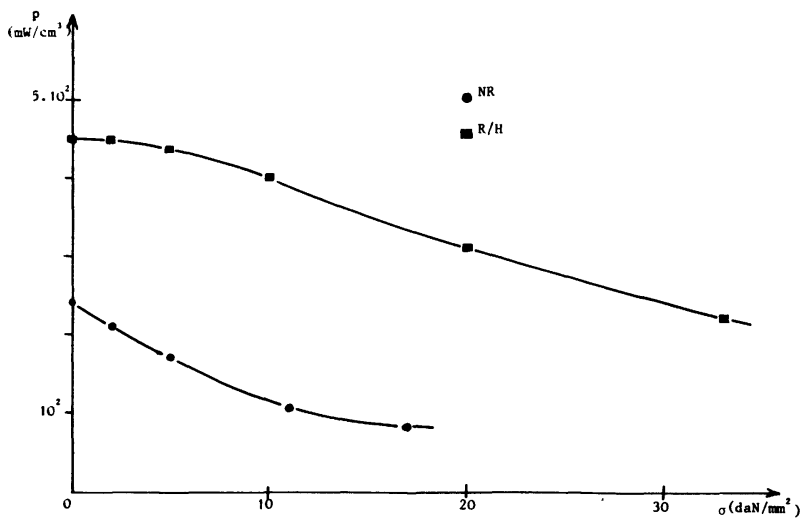

Fig. 11. - Pertes volumiques en fonction de $\sigma$. Ruban 2705 $M: B=0,5 \mathrm{~T}, f=10 \mathrm{kHz}$.

[Total losses versus $\sigma$. Ribbon $2705 \mathrm{M}: B=0.5 \mathrm{~T}$, $f=10 \mathrm{kHz}$.]

la diminution des pertes avec $\sigma$, en particulier dans le cas du matériau traité sous champ, infirme l'hypothèse selon laquelle la contrainte exercée n'a pas d'action sur la structure magnétique du ruban.

\section{Conclusion.}

Il apparaît, en premier lieu, que la caractérisation des échantillons entreprise sous champ sinusoïdal est mal adaptée dès qu'on désire effectuer une analyse fine des processus. Ce point est particulièrement important lorsque la perméabilité du matériau est très sensible à la contrainte exercée. En effet, l'application d'un effort s'accompagne alors d'une déformation notable du cycle d'hystérésis et donc d'une modification de la non-linéarité entre $B$ et $H$. Il s'ensuit que, le champ imposé restant sinusoïdal, le signal du flux à travers l'échantillon testé est déformé, ce qui se traduit par une variation du taux d'harmoniques. Les pertes dissipées, fonctions non linéaires de la vitesse de variation de l'induction, sont particulièrement sensibles à cette variation du taux d'harmoniques. De façon générale, les pertes mesurées sous champ sinusoïdal sont supérieures, ou égales, à celles obtenues sous flux (ou induction) sinusoïdal, pour une même valeur de l'induction maximale. L'étude effectuée sur l'alliage $2705 \mathrm{M}$ non recuit apparaît comme un exemple typique de ce problème. Il est clair qu'une meilleure compréhension de l'influence des efforts mécaniques nécessite des mesures sous flux sinusoïdal, afin de pouvoir effectuer des comparaisons plus significatives.

Cette étude permet, néanmoins, de relier les variations des pertes électromagnétiques en fonction de la contrainte et les modifications de la structure magnétique de l'échantillon. La fréquence de l'excitation magnétique étant un paramètre susceptible d'influencer cette structure [14], nous envisageons de compléter ce travail par la caractérisation de ces matériaux jusqu'à quelques centaines de kilohertz. De tels essais nécessitent, évidemment, la mise en œuvre de métho- 
des différentes de celles que nous avons utilisées [15], afin d'éliminer l'influence des effets capacitifs entre enroulements, susceptibles d'apparaître à haute fréquence.

Du point de vue de l'utilisateur, la présente étude montre qu'un effort exercé dans l'axe du champ magnétique peut conduire à une amélioration des performances dans le cas d'un matériau non traité et à une dégradation acceptable dans les autres cas. Par contre, le seul essai effectué en appliquant une contrainte perpendiculaire au champ fait apparaître une diminution notable de la perméabilité et une augmentation sensible des pertes. Ce point est crucial dans la mesure où il existe normalement, dans un rotor de machine électrique, des zones où les lignes d'induction sont perpendiculaires à la force centrifuge. Il est donc important de développer les études pour mieux caractériser ces effets.
Enfin, et dans la même optique, l'utilisation des alliages amorphes se heurte toujours aux problèmes de fragilisation provoquée par les traitements thermiques et de stabilité à long terme. Il n'est pas exclu que seuls les alliages non traités thermiquement puissent être utilisés pour la réalisation de machines de puissance massique élevée. Cependant, de nouveaux procédés de traitements thermiques [18], effectués à plus haute température et de durée moindre que ceux maintenant traditionnels, pourraient éviter cet écueil.

\section{Remerciements.}

Les auteurs remercient la D.R.E.T. qui a financé partiellement cette étude.

\section{Bibliographie}

[1] Nielsen, O. V., Nielsen, H. J. V., J. Magn. Magn. Mat. 22 (1980) 21.

[2] Dong, X. Z., Fernengel, W., Kronmuller, H., Appl. Phys. A. 28 (1982) 103.

[3] Tsumashima, S., Maehata, Y., Uchiyama, S., IEEE Trans. Mag. MAG 17 (1981) 3073.

[4] Luborsky, F. E., Livingston, J. D., IEEE Trans. Mag. MAG 18 (1982) 908.

[5] Конmoto, O., Fujishima, H., Ojima, T., IEEE Trans. Mag. MAG 16 (1980) 440.

[6] Nabais, A., Faugières, J. C., Rialland, J. F., BonneFILle, R., Revue Phys. Appl. 19 (1984) 1.

[7] Allied Corporation. Procedure for field annealing of soft magnetic Metglas alloys, Documentation commerciale, février 1981.

[8] Dong, X. Z., Kronmuller, H., Phys. Status Solidi (a) 70 (1982) 451

[9] Livingston, J. D., Morris, W. G., Luborsky, F. E., J. Appl. Phys. 53 (1981) 7837.
[10] Shilling, J. W., Morris, W. G., Osborn, M. L., IEEE Trans. Mag. MAG 14 (1978) 104.

[11] Livingston, J. D., Phys. Status Solidi (a) 56 (1979) 637.

[12] Fournier, P., Henry, M., Rev. Gen. Elect. (1983) 318.

[13] Hasegawa, R., Fish, G. E., Ramanan, V. R. V., Proc. 4th Int. Conf. Rapidly Quenched Metals Eds Masumoto and Suzuki, Japan Institute of Metals (1982).

[14] Washko, S. D., Osborn, M. L., VeERARaghaVEN, W. G., J. Appl. Phys. 52 (1981) 1899.

[15] DAN Y. Chen, Proc. IEEE, 69 (1981) 853.

[16] FuJimori, H., Magnetic anisotropy, p. 300, Amorphous Metallic alloys, F. E. Luborky ed. (Butterworths Monographs in Materials, Londres) 1983.

[17] Vasquez, M., Fernengel, W., Kronmüller, H., Phys. Status Solidi (a), 80513 (1983).

[18] TAUB, A. I., IEEE Trans. Mag, MAG 20 (1984) 564. 